\title{
Corylin sensitizes breast cancer cells to overcome tamoxifen resistance by regulating OAS1/miR-22-3p/SIRT1 axis
}

\author{
Li Che ${ }^{1}$, Hongru Yang², Daijie Wang² and Shourong Liu²区 \\ 'Department of Breast Disease, Luzhou People's Hospital, Luzhou, PR China; 2Department of Oncology and Hematology, Luzhou People's Hospi- \\ tal, Luzhou, PR China
}

Breast cancer $(\mathrm{BCa})$ is one of the leading causes of cancer-related death among women worldwide. At present, the clinical treatment with tamoxifen (TAM) is challenged by the development of drug resistance. To investigate the effect of corylin on TAM resistance in $\mathrm{BCa}$ cells, this study investigated the molecular mechanisms involving miRNA-mRNA targets modulated by corylin. The TAM-resistant MCF-7TR and T47DTR cell lines were generated, and it was found that corylin treatment reduced the cell viability of these cells significantly. Furthermore, OAS1 was validated to be highly expressed in TAM-resistant cells, while OAS1 knockdown sensitized MCF-7TR and T47DTR cells to TAM treatment. Meanwhile, OAS1 was also repressed by corylin treatment, indicating that OAS1 was a key regulator of corylin function. Through bioinformatic analysis, the tumor suppressive miRNA miR-22-3p was identified to directly target and inhibit OAS1. Moreover, corylin treatment up-regulated miR-22-3p expression, which thus down-regulated the OAS1 expression. Interestingly, OAS1 itself functioned as a miR-22-3p sponge to repress miR-22-3p expression. Further, SIRT1 was identified to be up-regulated in TAM-resistant cells and participated in the OAS1/ miR-22-3p regulatory axis via the miR-22-3p direct target. In conclusion, corylin sensitized TAM-resistant cells to TAM treatment by inhibiting OAS1 expression and modulating the OAS1/miR-22-3p/SIRT1 axis.

Keywords: corylin, OAS1, miR-22-3p, tamoxifen resistance, breast cancer

Received: 18 April, 2021; revised: 17 June, 2021; accepted: 15 July, 2021; available on-line: 03 November, 2021

घe-mail: shourong.liu@gmx.net

Abbreviations: BCa, breast cancer; MCF-7TR, MCF-7 tamoxifen resistant cell; TAM, tamoxifen; T47DTR, T47D tamoxifen resistant cell

\section{INTRODUCTION}

Breast cancer $(\mathrm{BCa})$ is one of the leading causes of cancer-related deaths among women worldwide (Siegel et al., 2018). Tamoxifen (TAM), the estrogen antagonist, is widely used as a part of chemotherapy for luminal A type $\mathrm{BCa}$ patients with positive estrogen receptor $(\mathrm{ER}+)$ (Lim \& Winer, 2011; Ignatiadis \& Sotiriou, 2013). The application of TAM reduces the mortality to $31 \%$ (Hultsch et al., 2018). Nevertheless, clinical case reports reveal that around $30 \%$ of patients develop intrinsic or acquired resistance to TAM, leading to a lower TAM efficiency and the challenges for $\mathrm{BCa}$ treatment (Cao et al., 2019). Thus, it is necessary to elucidate the potential molecular mechanism of TAM resistance and to provide promising approaches to overcome TAM resistance in BCa treatment.
Many studies have reported that various compounds extracted from different Chinese herbal medicines can prevent cancer progression and may be used in clinical treatment (Qiu et al., 2020). In this study, corylin, the flavonoid compound extracted and purified from Psoralea corylifolia L. (Fabaceae), was studied for its role in TAM resistance during $\mathrm{BCa}$ treatment. As revealed by the previous studies, corylin exhibits anti-tumor and antiinflammation activities in cancers such as hepatocellular carcinoma (HCC) (Lu et al., 2018). However, none of the existing studies focuses on the effect of corylin on TAM resistance, and the molecular mechanism of corylin function remains unclear. Here, we demonstrated that corylin sensitized the TAM-resistant MCF-7 and T47D cells to TAM treatment.

The 2'-5' oligoadenylate synthetase (OAS) family consists of the interferon (IFN)-induced antiviral enzymes, which contains four members including OAS1, OAS2, OAS3 and OASL (Hovnanian et al., 1998; Kristiansen et al., 2011). According to previous studies, the OAS family is involved in different diseases and plays important roles in biological functions like cell growth, apoptosis and gene regulation (Choi et al., 2015; Hancks et al., 2015). In the present study, OAS1 expression increased in TAMresistant $\mathrm{BCa}$ cells, and corylin treatment reduced its expression. In addition, microRNA-22 (miR-22-3p), an extensively studied miRNA that played a role as a tumor suppressor in cancer progression, was firstly identified to target OAS1 directly. MiRNA is a type of non-coding RNA containing 18-22 nucleotides, which exhibits diverse activities in diseases and plays interactive roles in the functions of a variety of extracted compounds. miR-22-3p has been confirmed as a tumor suppressive miRNA in different cancers (Tian et al., 2021; Yang et al., 2021). It is also a senescence related miRNA that represses cancer progression by inducing cellular senescence via targeting multiple genes including SIRT1, p21 and MDC1 (Tsuchiya et al., 2011; Xu et al., 2011; Lee et al., 2015). Therefore, the potential molecular mechanisms related to the miR-22-3p/OAS1 axis regulated by corylin were investigated in this study.

\section{MATERIALS AND METHODS}

\section{Materials}

Corylin (PHL83287) was purchased from SigmaAldrich. The specific OAS1 and SIRT1 siRNAs were designed and synthesized by Sangon (Shanghai, China). All cell culture materials including 6-well plates and T75 flasks were purchased from Corning (USA). The primary antibody against OAS1 was purchased from Abcam 
(USA). The xCELLigence system was obtained from Agilent (USA). SYBR Green mix for q-PCR was purchased from QIAGEN (Germany). Overexpression plasmid pcD-OAS1 and pcD-SIRT1 was designed and synthesized at the Beijing Genomics Institute.

\section{Cell culture and treatment}

For corylin preparation, corylin was dissolved in DMSO to obtain a stock concentration of $50 \mathrm{mM}$, then corylin stock solution was diluted with HBSS (Gibco, USA) to prepare a working solution at the concentration of $1 \mathrm{mM}$. The final concentration of DMSO in the medium was less than $0.02 \%$ to avoid the side effect of DMSO on cell viability. HBSS was served as a negative control to corylin treatment. Before testing corylin effect, cells were pre-treated with corylin for $12 \mathrm{~h}$. The Estrogen receptor positive $(\mathrm{ER}+)$ and tamoxifen (TAM) sensitive human breast cancer cell lines MCF-7 (HTB-22) and T47D (HTB-133) were purchased from the American Type Culture Collection (ATCC; Manassas, VA, USA) and were cultured in Dulbecco's modified Eagle's medium (DMEM; Invitrogen, Carlsbad, CA, USA) containing 10\% fetal bovine serum (FBS; Hyclone, Logan, UT, USA), $100 \mathrm{U} / \mathrm{mL}$ penicillin and $100 \mu \mathrm{g} / \mathrm{ml}$ streptomycin in a humidified atmosphere of $95 \%$ air and $5 \% \mathrm{CO}_{2}$ at $37^{\circ} \mathrm{C}$. For TAM resistant MCF-7 cell (MCF-7TR) and TAM resistant T47D cell (T47DTR) generation, parental MCF-7 and T47D cells were continuously treated and exposure to $1 \mu \mathrm{M}$ TAM (Sigma-Aldrich, USA) for at least 6 months, and then obtained TAM resistant cells were cultivated and maintained in DMEM medium containing $1 \mu \mathrm{M}$ TAM.

\section{Transfection}

siRNAs and miRNA mimics were transfected to the cells with Lipofectamine RNAiMAX reagent (Thermo Fisher, USA). Plasmids were transfected to the cells with Lipofectamine 2000 (Thermo Fisher, USA). Cells were seeded into 6 -well plate at the density of $5 \times 10^{5}$ cells/well and cultivated overnight to over $70 \%$ confluence before transfection. Diluted siRNAs, miRNA mimics/inhibitor or plasmid with OptiMEM medium (Gibco, USA) to total volume $150 \mu \mathrm{l} /$ well. Made $150 \mu \mathrm{l}$ LipofectamineOptiMEM mixture for each sample and incubated the mixture with RNA or plasmid-OptiMEM dilution for 5 min at room temperature. The ratio of Lipofectamine and RNAs (plasmid) was 2:1 (v/v). The final concentrations of RNAs (siRNA, miRNA mimics/inhibitor) and plasmids were $25 \mathrm{nM}$ and $1 \mu \mathrm{g} / \mathrm{ml}$, respectively. Incubated mixture was then added to the cells and cultivated for 48 h. OAS1 overexpression vector (pcD-OAS1) was designed and constructed by Beijing Genomics Institute (Beijing, China). OAS1 and SIRT1 siRNAs were purchased from QIAGEN (Germany). miR-22-3p mimics and inhibitor were purchased from Biomics (Jiangsu, China).

\section{Cell viability assessment}

Breast cancer cells were seeded into 96-well plates at the density of $3 \times 10^{3}$ cells/well and cultured overnight. Before experiment, the background impedance of Eplate 16 (Agilent, USA) was measured by adding $100 \mu \mathrm{l}$ cell culture media at room temperature. Then $100 \mu \mathrm{l}$ cell suspension containing all cells of each well in 96-well plate was added into $100 \mu \mathrm{l}$ media in E-plate. After incubating for $1 \mathrm{~h}$, E-plate 16 was inserted to Xcelligence device (RTCA DP, Agilent, USA). Impedance was monitored every 60 minutes for a period of up to 48 hours.

The relative changes of electrical impedance caused by cell adhesion on surface were calculated and represented by a unitless parameter CI, which was calculated according to the formula: $\mathrm{CI}=(\mathrm{Zi}-\mathrm{Z} 0) / 15$, where $\mathrm{Zi}$ represents the impedance at an individual point of time during the experiment and $\mathrm{ZO}$ is the impedance at the start of the experiment. Three different frequencies (10, 25 or $50 \mathrm{kHz}$ ) and a specific time was used to measure the impedance based on the RTCA DP Instrument Operator's Manual. Meanwhile, cells were seeded into 96well plate parallelly in triplicates and cell numbers were counted at the indicated time points.

\section{Colony formation assay}

MCF-7 and T47D cells with $10 \mu \mathrm{M}$ corylin treatment were seeded into 6 -well plate at the density of $3 \times 10^{3}$ cells/well. Cells were incubated for 3 weeks until cell colony is visible to the naked eye. Cells were washed twice with PBS after discarding media. Methanol at $800 \mu \mathrm{l} /$ well was used to fix cells for $30 \mathrm{~min}$ and then cells were stained with $0.5 \%$ crystal violet for $30 \mathrm{~min}$. After staining, cells were washed with PBS 3 times. Images were obtained through taking pictures with a digital camera (Nikon, Japan) and cell numbers were quantified with ImageJ software.

\section{Flow Cytometry}

Cell cycle was detected by using propidium iodide (PI, BD Biosciences, No.51-66211E, $1.5 \mathrm{mg} / \mathrm{ml}$ ). Cells were seeded in 6 -well plates at a density of $2 \times 10^{5}$ cells/well with different treatments. Next, cells were resuspended in binding buffer and stained with $10 \mu \mathrm{l}$ PI for $10 \mathrm{~min}$ at room temperature (avoiding light). Then, the stained cells were analyzed with a flow cytometer (BD Biosciences) and DNA contents were quantified. Each sample was tested in triplicate.

\section{Quantitative Real-time PCR}

Total RNA was isolated with High Pure RNA Isolation Kit (Roche, Switzerland) based on the instruction from the manufacturer. cDNAs were obtained through the total RNA (100 ng) reverse transcription with verso cDNA Synthesis Kit (Thermo Fisher, USA) based on the product instruction. SYBR ${ }^{\mathrm{TM}}$ Green PCR Master Mix (Thermo Fisher, USA) was used for q-PCR reaction in $15 \mu \mathrm{l}$ system. Q-PCR reaction cycle: $95^{\circ} \mathrm{C} 30 \mathrm{~s} ; 95^{\circ} \mathrm{C}$ $30 \mathrm{~s}, 65^{\circ} \mathrm{C} 30 \mathrm{~s}, 72^{\circ} \mathrm{C} 30 \mathrm{~s}$ for 40 cycles. Expression was normalized using the detection of GAPDH using the $2^{\wedge}(-\Delta \Delta \mathrm{Ct})$ method (Livak \& Schmittgen, 2001). Primers used in q-PCR were listed in Table 1.

\section{Western blot}

Cells were lysed with RIPA buffer, and the lysate was quantified with BCA kit (Thermo Fisher, USA) according to the manufacturer's instructions. Total $40 \mu \mathrm{g}$ protein

\begin{tabular}{lc} 
Table 1. Primers & used in $\mathbf{q}-P C R$ analysis $\left(5^{\prime}\right.$ to $\left.\mathbf{3}^{\prime}\right)$. \\
\hline Primers & Sequence \\
\hline OAS1 forward & TGTCCAAGGTGGTAAAGGGTG \\
\hline OAS1 reverse & CCGGCGATTAACTGATCCTG \\
\hline SIRT1 forward & TAGCCTTGTCAGATAAGGAAGGA \\
\hline SIRT1 reverse & ACAGCTTCACAGTCAACTTGT \\
\hline
\end{tabular}


was loaded for electrophoresis in 10\% SDS-PAGE gel. After electrophoresis, protein was transferred onto PVDF membrane. PVDF membrane containing protein was blocked by $5 \%$ skim milk dissolved in TBS for $30 \mathrm{~min}$ at room temperature. Primary antibodies OAS1 (14955-1AP, Proteintech, USA) and $\beta$-actin (Wanlei, China) were diluted into 5\% BSA dissolved in TBS at 1:2000. Then, PVDF membrane was incubated with diluted primary antibodies for overnight at $4^{\circ} \mathrm{C}$. Secondary antibodies were diluted into 5\% skim milk dissolved in TBS (1:10000) and incubated with membranes for $1 \mathrm{~h}$ to $1.5 \mathrm{~h}$ at room temperature while gently shaking. Enhanced chemiluminescence (ECL) system was employed for imaging.

\section{RNA immunoprecipitation}

RNA immunoprecipitation was performed as previously described (Peritz et al., 2006). AGO2 (anti-AGO2, 1:50, No. 2897s, Cell Signaling, USA) was immunoprecipitated by using $25 \mu \mathrm{l}$ protein $\mathrm{A} / \mathrm{G}$ agarose. Trizol agent was used to extract total RNA after immunoprecipitation. qPCR was used to measure levels of miR-223p, OAS1 and SIRT1.

\section{Bioinformatic analysis}

Expression and clinical data of GSE148878 was downloaded from National Center for Biotechnology Information Gene Expression Omnibus (www.ncbi.nlm.nih.gov/ geo). Up-regulated genes were enriched in Hallmark gene sets through Molecular Signatures Database (MSigDB, https://www.gsea-msigdb.org/gsea/msigdb/index.jsp).

miRNA targets prediction was performed via ENCORI (http://starbase.sysu.edu.cn/panCancer.php).

\section{Statistics}

All data are presented as mean \pm S.D. values. Analysis was achieved by two-way analysis of variance and $t$-test.
$P$ values of $<0.05\left({ }^{*} p\right)$ or $<0.01(* * p)$ were considered statistically significantly different.

\section{RESULTS}

\section{Corylin inhibited the development of ER+ BCa cells}

To verify the effect of corylin on the development of ER positive $(\mathrm{ER}+) \mathrm{BCa}$ cells, different concentrations $(0-20 \mu \mathrm{M})$ of corylin were used to test the proliferation rates of MCF-7 and T47D cells. As shown in Fig. 1A and $1 \mathrm{~B}$, the proliferation rates were measured using the xCELLigence Real-Time Cell Analysis system. As a result, corylin significantly inhibited the proliferation of MCF-7 and T47D cells in a concentration-dependent manner. The half-maximal inhibitory concentrations (IC50) of corylin on MCF-7 and T47D cells were 10.31 $\mu \mathrm{M}$ and $13.48 \mu \mathrm{M}$, respectively. When MCF-7 and T47D were treated with $10 \mu \mathrm{M}$ corylin, the proliferation rates were significantly repressed (Fig. 1C). Therefore, $10 \mu \mathrm{M}$ corylin was used in the following experiments. In addition, colony formation assay also indicated that corylin suppressed the colony formation capacity of BCa cells (Fig. 1D). To further explore whether the observed phenomenon was related to cell cycle, flow cytometric analysis was performed. After corylin treatment, the cell cycle of MCF-7 and T47D cells were arrested in G2/M phase compared with control group (Fig. 1E).

\section{Corylin reduced TAM resistance}

Next, we generated the TAM-resistant MCF-7 and T47D cells (MCF-7TR and T47DTR, respectively). It was observed that, compared with parental cells treated with TAM, MCF-7TR and T47DTR cells acquired TAM resistance as the cell viability was maintained at a high level (Fig. 2A and 2B). The IC50 values of TAM
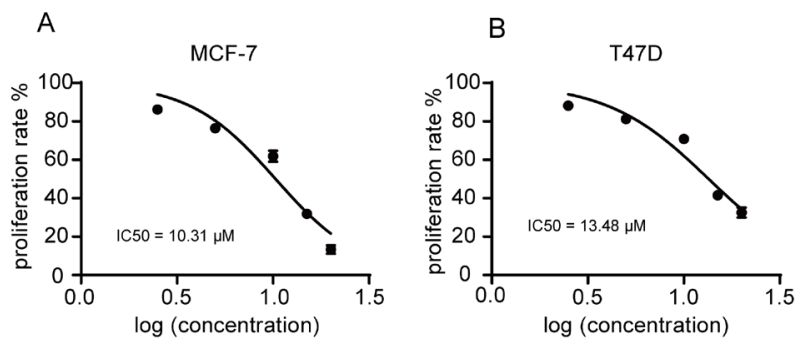

C

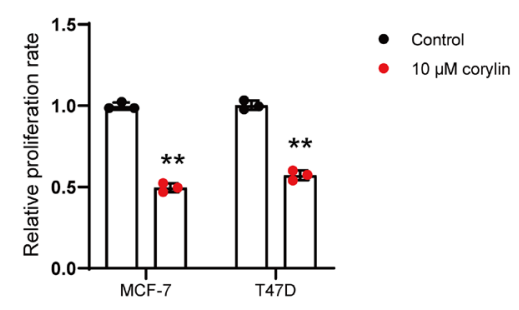

D

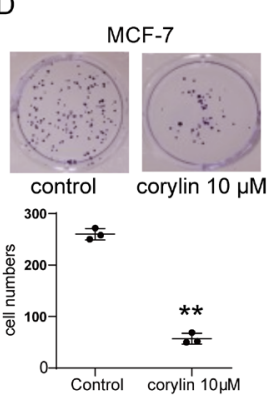

T47D

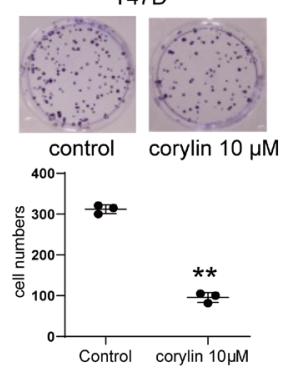

E

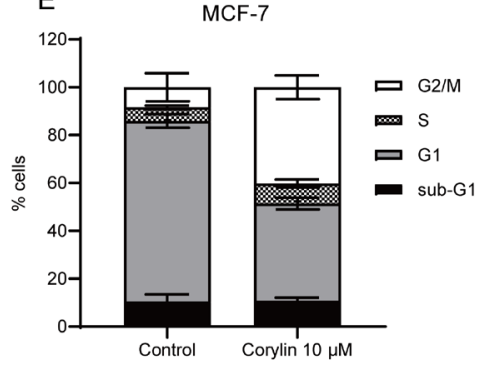

T47D

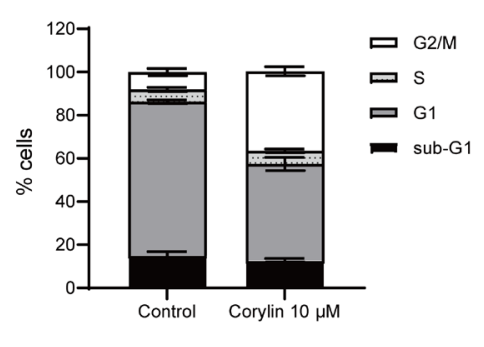

Figure 1. Corylin inhibits development of ER+ breast cancer cells

(A) The effect of corylin with different concentrations on proliferation of MCF-7. (B) The effect of corylin with different concentrations on proliferation of T47D. (C) $10 \mu \mathrm{M}$ corylin was selected to test the effect on cell proliferation rate of MCF-7 and T47D cells (** $p<0.01$ ). (D) The effect of $10 \mu \mathrm{M}$ corylin on colony formation capacity of MCF-7 and T47D tested by colony formation assay (**p<0.01). (E) The effect of $10 \mu \mathrm{M}$ corylin on cell cycle of MCF-7 and T47D tested by Flow Cytometry. 

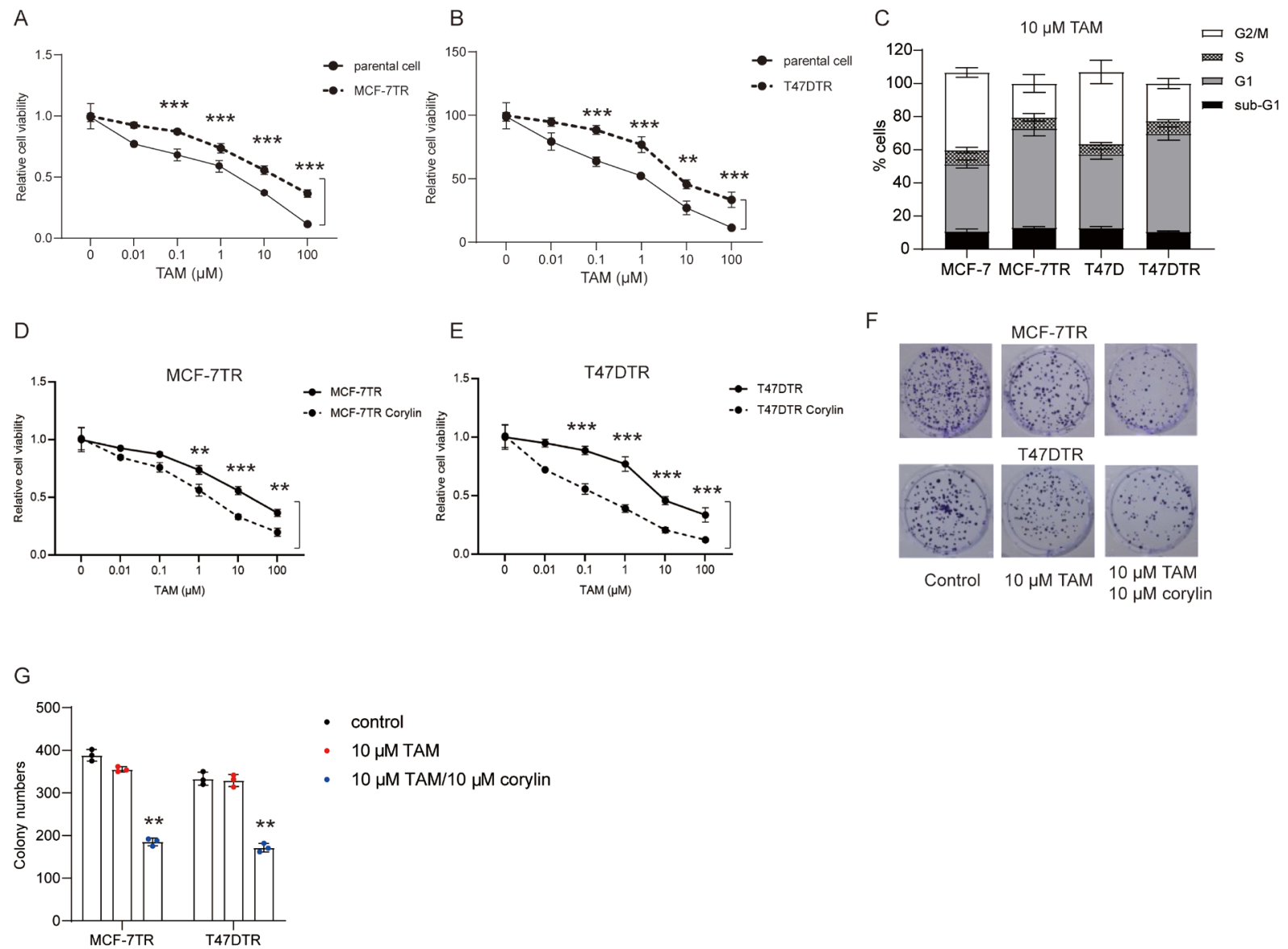

Figure 2. Corylin reduces TAM resistance

(A) Cell viability of MCF-7 and MCF-7TR were measured by xCELLigence system with TAM treatment. Cell viability was compared between parental cells and TAM resistant cells at different TAM concentration $\left({ }^{* *} p<0.001\right)$. (B) Cell viability of T47D and T47DTR were measured by xCELLigence system with TAM treatment. Cell viability was compared between parental cells and TAM resistant cells at different TAM concentration $\left({ }^{* *} p<0.01,{ }^{* * *} p<0.001\right)$. (C) The effect of $10 \mu M$ TAM on cell cycle of MCF-7, MCF-7TR, T47D and T47DTR were tested by Flow Cytometry. Corylin reduced cell viability of MCF-7TR (D) and T47DTR (E) when treated with TAM (** $p<0.01$, *** $p<0.001)$. (F) $10 \mu \mathrm{M}$ corylin reduced proliferation of MCF-7TR and T47DTR treated with TAM. The colony numbers were quantified by Image J presented in $(\mathbf{G})\left({ }^{* *} p<0.01\right)$.

on parental MCF-7 and T47D cells were $9.24 \mu \mathrm{M}$ and $7.83 \mu \mathrm{M}$, respectively, while those on MCF-7TR and T47DTR cells were $15.81 \mu \mathrm{M}$ and $12.36 \mu \mathrm{M}$, respectively. Flow cytometry results also showed that the percentage of apoptotic cells decreased in MCF-7TR and T47DTR cells treated with $10 \mu \mathrm{M}$ TAM compared with that in parental cells as cells in G2/M phase (Fig. 2C). After combined treatment of $10 \mu \mathrm{M}$ corylin with TAM, the cell viability of MCF-7TR and T47DTR cells significantly reduced (Fig. 2D and 2E). At last, colony formation assay illustrated that corylin suppressed the colony formation capacity of TAM-resistant cells, and less colonies were formed (Fig. 2F and 2G).

\section{OAS1 was regulated by corylin in TAM-resistant BCa cells}

To further clarify the molecular mechanisms involved in the corylin effect, differentially expressed genes (DEGs) were analyzed. Gene expression data were obtained from the GEO datasets (GSE148878), where RNA-seq was performed in TAM-sensitive and TAM-resistant MCF-7 cells. Gene expression levels in TAM-resistant MCF-7 cells were compared with those in parental MCF-7 cells. There were altogether 73 up-regulated DEGs with the fold change (FC) over 1.5 whereas 10 down-regulated DEGs with FC less than 0.5 (Fig. 3A).
Most of the up-regulated DEGs $(n=11)$ were enriched in the gene set of cytokine signaling (Fig. 3B), listed in Table 2. Next, q-PCR was carried out to test the DEGs enriched in the gene set of cytokine signaling. As shown in Fig. 3C, OAS1 was over-expressed in MCF-7TR and T47DTR cells, as confirmed by q-PCR. In addition,

Table 2. 11 up-regulated genes enriched in gene set of cytokine signaling.

\begin{tabular}{ll}
\hline Gene symbol & Description \\
\hline OAS1 & $2^{\prime}$-5'-oligoadenylate synthetase 1 \\
\hline IFIT1 & $\begin{array}{l}\text { interferon induced protein with tetratricopepti- } \\
\text { de repeats } 1\end{array}$ \\
\hline IFI35 & interferon induced protein 35 \\
\hline IFI27 & interferon alpha inducible protein 27 \\
\hline XAF1 & XIAP associated factor 1 \\
\hline GBP3 & guanylate binding protein 3 \\
\hline LYN & LYN proto-oncogene \\
\hline CD36 & CD36 molecule \\
\hline PSMB9 & proteasome 20S subunit beta 9 \\
\hline IL15 & interleukin 15 \\
\hline SOX2 & SRY-box transcription factor 2 \\
\hline
\end{tabular}


A

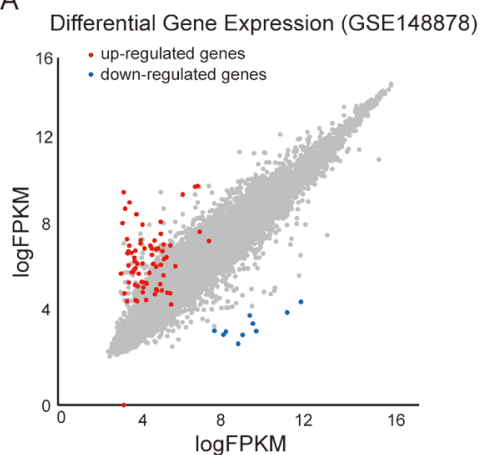

D

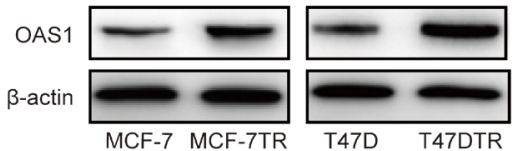

G

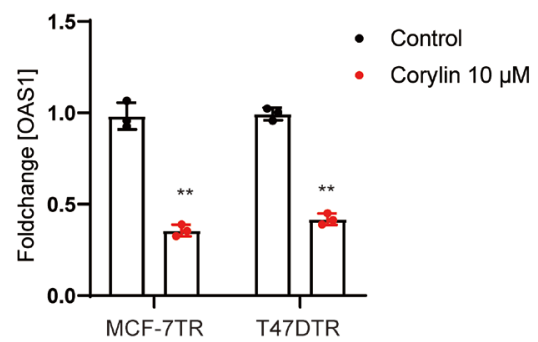

$\mathrm{E}$
$B$

Enriched Gene sets for 73 up-regulated genes

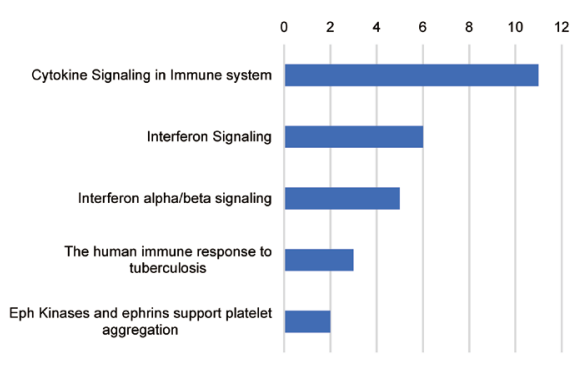

C

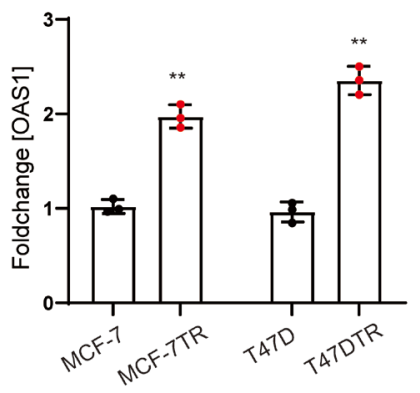

\section{$\mathrm{F}$}

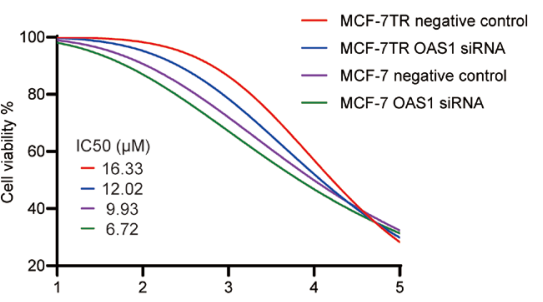

$\log ($ TAM concentration, $\mathrm{nM}$ )

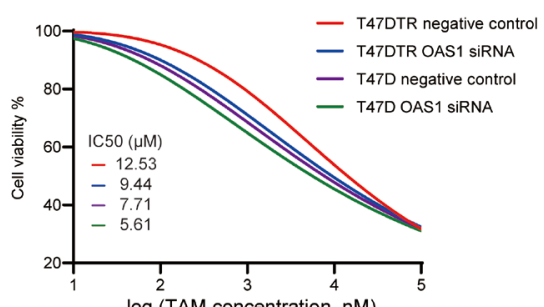

$\log ($ TAM concentration, nM)

Figure 3. OAS1 is overexpressed and regulated by corylin in TAM resistant breast cancer cells

(A) GSE148878 was analyzed to obtain differential expressed genes in TAM resistant cells. (B) 73 up-regulated genes were analyzed and enriched in different gene sets. (C) q-PCR tested OAS1 highly expressed in MCF-7TR and T47DTR cells (**p<0.01). (D) Western blot tested OAS1 protein highly expressed in MCF-7TR and T47DTR cells. (E) and (F) Cell viability of parental and TAM resistant MCF-7 and T47D cells were tested with OAS1 silencing and TAM treatment. IC50 values were labeled in each figure. (G) The effect of $10 \mu \mathrm{M}$ corylin treatment on OAS1 mRNA expression in MCF-7TR and T47DTR cells tested by q-PCR $\left({ }^{* *} p<0.01\right)$. (H) The effect of $10 \mu \mathrm{M}$ corylin treatment on OAS1 protein expression in MCF-7TR and T47DTR cells tested by western blot.

OAS1 protein level increased compared with that in parental MCF-7TR and T47TR cells (Fig. 3D). Thereafter, the specific siRNA pools of OAS1 were transfected into TAM-resistant cells. According to our results, knocking down of OAS1 significantly reduced cell viability of both parental and TAM-resistant cells. The IC50 of TAM was lower when OAS1 was silenced in both parental and TAM-resistant cells, as indicated in Fig. 3E, 3F. Notably, parental cells with OAS1 silencing showed the highest sensitivity to TAM treatment. In the following step, the effect of corylin on OAS1 was measured. In TAM-resistant cells, corylin treatment remarkably inhibited OAS1 at both mRNA and protein levels (Fig. $3 \mathrm{G}$ and $3 \mathrm{H}$ ).

\section{Corylin reduced TAM resistance by regulating the miR- 22-3p/OAS1 targeting}

We further investigated the underlying mechanism by which corylin and OAS1 exerted their biological functions in TAM-resistant BCa cells. By using the online bioinformatics tools such as TargetScan 7.0 and ENCORI, miR-22-3p was predicted to target OAS1 directly (Fig. 4A). Indeed, results of luciferase assay revealed that the reporter vector containing the wild type sequences of OAS1 target (pGL3-OAS1 WT) showed significantly suppressed luciferase activity compared with that of mutant vector (pGL3-OAS1 W'T) after miR-22-3p mimics transfection (Fig. 4B). In addition, miR-22-3p was validated to have a lower expression level in TAM-resistant cells than that in parental cells (Fig. 4C). Corylin treatment induced miR-22-3p expression in MCF-7TR and T47DTR cells (Fig. 4D) compared with HBSS treatment as a control, while miR-22-3p mimics inhibited OAS1 expression at mRNA (Fig. 4E) and protein levels (Fig. 4F). Interestingly, OAS1 knockdown also increased miR-22-3p expression in TAM-resistant cells (Fig. 4G), which indicated that OAS1 itself sponged the endogenous miR-22-3p and subsequently affected the downstream targets of miR-22-3p.

\section{Corylin reduced TAM resistance by regulating the OAS1/miR-22-3p/SIRT1 axis}

Since OAS1 functions as a sponge of miR-22-3p, it is intriguing to reveal the potential downstream target. SIRT1 plays crucial roles in many biological processes such as aging, apoptosis and tumor development. It is a well-studied miR-22-3p target in several cancer types (Tian et al., 2021; Yang et al., 2021). Although SIRT1 was not found to be up-regulated in TAM-resistant MCF-7 cells in the GSE148878 dataset, SIRT1 was confirmed to be over-expressed in our TAM-resistant cells, as verified by q-PCR (Fig. 5A). Corylin treatment inhibited SIRT1 expression in TAM-resistant cells (Fig. 5B). Furthermore, 


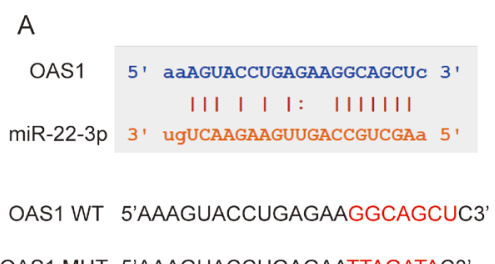

OAS1 MUT 5'AAAGUACCUGAGAATTAGATAC3'

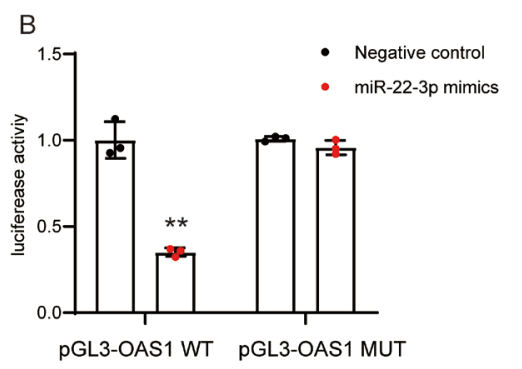

D

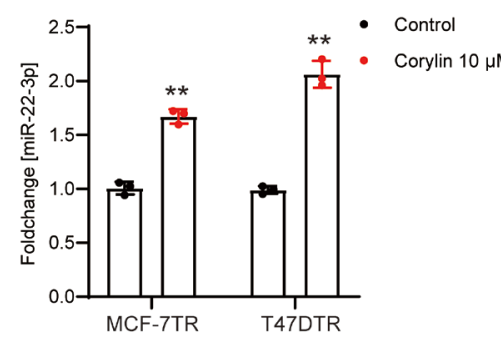

G

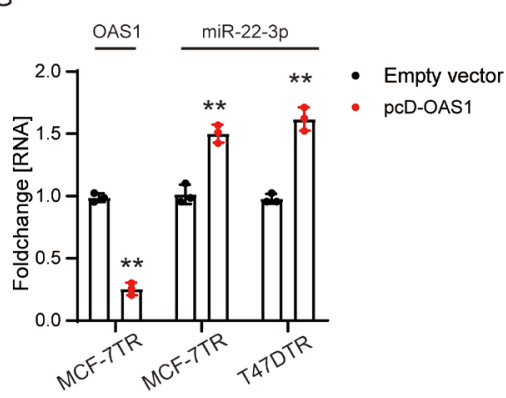

E

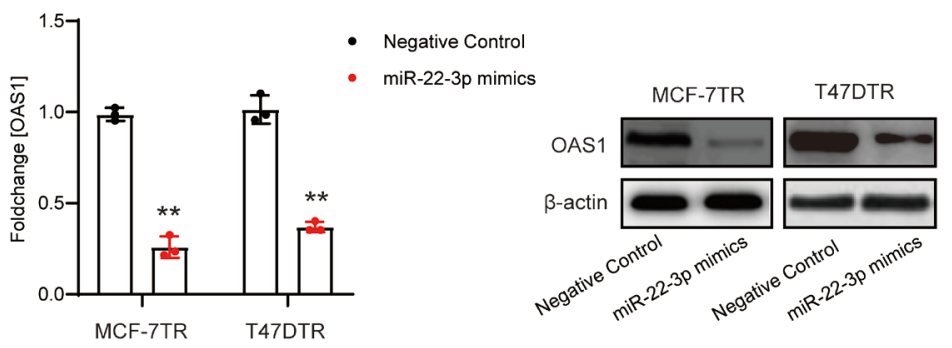

F

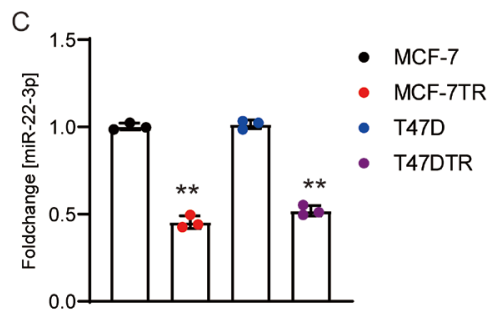

Figure 4. Corylin reduces TAM resistance by regulating miR-22-3p/OAS1 targeting

(A) OAS1 was predicted as a direct target of miR-22-3p by online informatic tool (ENCORI). (B) Luciferase assay tested the effect of miR$22-3 p$ mimics on luciferase activity of reporter system $(* * p<0.01)$. (C) miR-22-3p expression was reduced in TAM resistant cells analyzed by q-PCR (** $p<0.01)$. (D) $10 \mu \mathrm{M}$ corylin increased miR-22-3p expression in MCF-7TR and T47DTR $\left({ }^{* *} p<0.01\right)$. miR-22-3p reduced OAS1 expression in MCF-7TR and T47DTR in mRNA (E) and protein level (F). $\left.{ }^{* *} p<0.01\right)$. (G) OAS1 knocking down increased miR-22-3p expression $\left({ }^{* *} p<0.01\right)$.

OAS1 knockdown decreased SIRT1 expression (Fig. 5C) but SIRT1 knockdown (Fig. 5D) did not affect OAS1 expression (Fig. 5E). In addition, when miR-22-3p was continuously repressed by miR-22-3p inhibitor, SIRT1 expression still stayed at a high level after using $10 \mu \mathrm{M}$ corylin, which indicated that miR-22-3p was the critical intermediator between corylin-reduced OAS1 and SIRT1 (Fig. 5F). Next, RNA immunoprecipitation assay showed that both OAS1 and SIRT1 were enriched by AGO2 antibody after miR-22-3p mimics transfection (Fig. 5G), indicating the interactions among OAS1, miR-22-3p and SIRT1. To confirm whether miR-22-3p and SIRT1 were essential for the effect of corylin on reducing TAM resistance, we transfected pcD-SIRT1 and treated TAMresistant cells with corylin simultaneously. As a result, the ectopic expression of SIRT1 largely abrogated the effect of corylin on the cell viability of both MCF-7TR and T47DTR cells treated with $10 \mu \mathrm{M}$ TAM (Fig. 5H). Furthermore, miR-22-3p inhibitor was also employed together with corylin and TAM treatment. Similarly, miR22-3p inhibition rescued the cell viability of MCF-7TR and T47DTR cells, which was repressed by corylin and TAM treatment (Fig. 5I). Collectively, the OAS1/miR$22-3$ p/SIRT1 regulatory axis was critical for the effect of corylin on reducing TAM resistance in MCF-7TR and T47DTR cells.

\section{DISCUSSION}

$\mathrm{BCa}$ is the most common invasive cancer in women around the world and is the second leading cause of cancer-related deaths for women only second to lung cancer (Siegel et al., 2018). In clinical treatment, TAM is widely applied for BCa treatment, especially for the prevention of early precancerous lesion and luminal A type with positive ER (Ignatiadis \& Sotiriou, 2013). However, the development of intrinsic or acquired TAM resistance makes it challenging to treat $\mathrm{BCa}$ with TAM. In our study, we firstly tested the effect of corylin, the main compound extracted from Psoralea corylifolia L., on sensitizing the TAM-resistant MCF-7 and T47D cells to TAM. Natural products extracted from plants or Chinese herbal medicines have been identified to have important activities, such as anti-tumor and anti-inflammation effects (Qiu et al., 2020). However, limited studies have investigated the effects of those compounds on the prevention of TAM resistance. In this study, we revealed that corylin played a role in preventing TAM resistance 


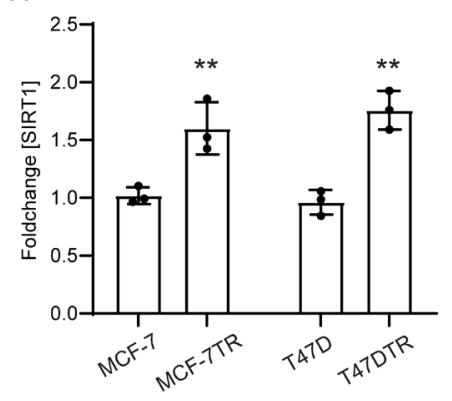

D

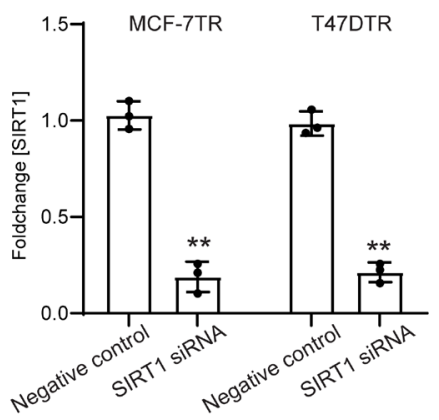

G

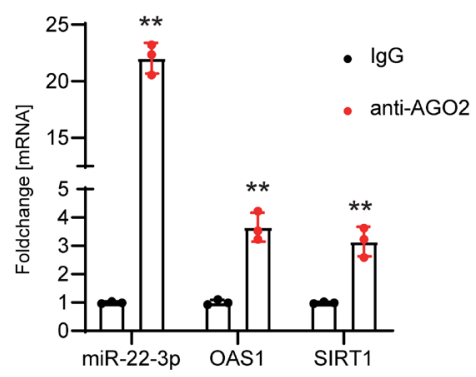

B
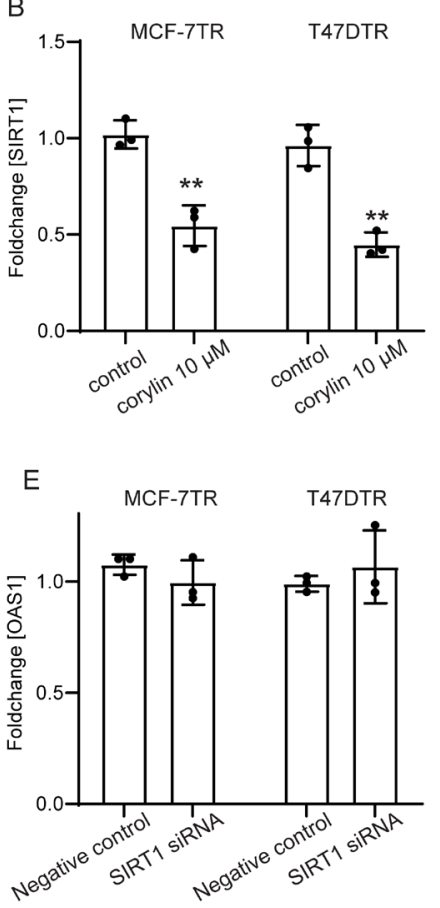

$\mathrm{H}$

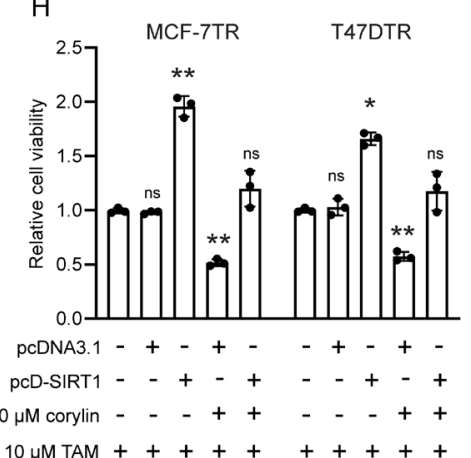

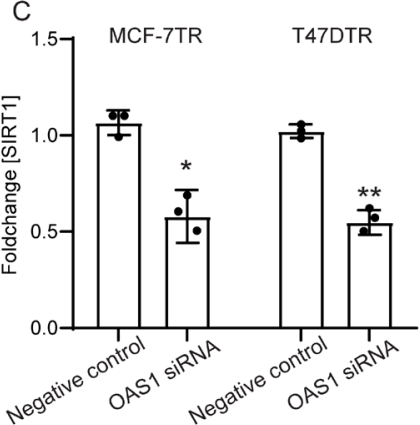

F

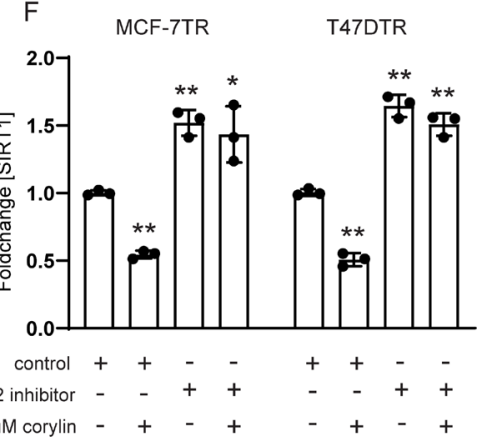

I

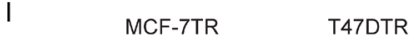

Figure 5. Corylin reduces TAM resistance by regulating OAS1/miR-22-3p/SIRT1 axis

(A) q-PCR tested SIRT1 highly expressed in MCF-7TR and T47DTR cells $\left({ }^{* *} p<0.01\right)$. (B) $10 \mu \mathrm{M}$ corylin reduced SIRT1 expression in MCF-7TR and T47DTR tested by q-PCR $\left({ }^{* *} p<0.01\right)$. (C) OAS1 knocking down decreased SIRT1 expression $\left({ }^{*} p<0.05,{ }^{* *} p<0.01\right)$. (D) The efficiency of SIRT1 siRNA in MCF-7TR and T47TR cells (**p<0.01). (E) SIRT1 knocking down cannot affect OAS1 expression tested by q-PCR. (F) The effect of co-treatment of miR-22-3p inhibitor and $10 \mu \mathrm{M}$ corylin on SIRT1 expression tested by $q-P C R$ analysis $\left({ }^{*} p<0.05,{ }^{* *} p<0.01\right)$. (G) The interaction among OAS1, miR-22-3p and SIRT1 was confirmed by RNA immunoprecipitation assay $\left({ }^{* *} p<0.01\right)$. (H) The effect of ectopic SIRT1 expression and $10 \mu \mathrm{M}$ corylin treatment on cell viability of MCF-7TR and T47DTR cells (ns: not significant, ${ }^{*} p<0.05$, ${ }^{* *} p<0.01$ ). (I) The effect of miR-22-3p inhibition, $10 \mu \mathrm{M}$ corylin and $10 \mu \mathrm{M}$ TAM treatment on cell viability of MCF-7TR and T47DTR cells (ns: not significant, $\left.{ }^{*} p<0.05,{ }^{* *} p<0.01\right)$.

through modulating OAS1 related to miRNA-mRNA targeting.

To verify the exact molecular mechanism of corylin function, we employed the GEO dataset GSE148878 to obtain DEGs in TAM-resistant BCa cells. Among those most significantly up-regulated genes, OAS1 was found to be highly expressed in MCF-7TR and T47DTR cells. Indeed, OAS1 and other OAS family members are correlated with the poor prognostic outcomes of $\mathrm{BCa}$ (Zhang \& Yu, 2020). The high OAS1 expression is associated with a low survival rate in luminal A type cancers (Zhang \& Yu, 2020). In addition, the OAS family members are also involved in neutrophil function that regulates the metastatic or recurrent potential of circulating tumor cells (Szczerba et al., 2019). Here, OAS1 was identified to be down-regulated after corylin treatment and was also the direct target of miR-22-3p. Previous studies have illustrated that miR-22-3p usually plays a role of tumor suppressor in different cancer types, including colorectal cancer (CRC) (Sun et al., 2019), ovarian cancer (Zhang et al., 2020), and cervical cancer (Wang et al., 2020), where miR-22-3p inhibits metastasis, attenuates inflammation and induces apoptosis. Interestingly, in our study, OAS1 and miR-22-3p affected each other, since miR-22-3p mimics inhibited OAS1 expression; by contrast, OAS1 knockdown or corylin treatment increased miR-22-3p expression in TAM-resistant cells.

Furthermore, the downstream targets of miR-22-3p were also investigated here. SIRT1 was found to be highly expressed in MCF-7TR and T47DTR cells, even though the GEO dataset did not indicate its overexpression. Corylin treatment inhibited SIRT1 expression in TAM-resistant cells. SIRT1 is previously proved as a direct target of miR-22-3p, and the targeted regulation is involved in cancer progression like metastasis and cellular senescence (Xu et al., 2011). Therefore, SIRT1 
was taken into consideration in this study to elucidate the corylin function. In this research, changes in OAS1 expression affected SIRT1 expression, but SIRT1 did not regulate OAS1, which indicated that OAS1 was located in the upstream of the whole regulatory axis. Interestingly, miR-22-3p inhibition abrogated the effect of corylin on SIRT1 expression, suggesting that miR-22$3 \mathrm{p}$ mediated the function of corylin depending on the OAS1/miR-22-3p/SIRT1 axis. In addition, both SIRT1 overexpression and miR-22-3p repression largely abrogated the effect of corylin on reducing the cell viability of TAM-resistant cells, which further illustrated that the downstream miR-22-3p/SIRT1 target was necessary for corylin function. Furthermore, SIRT1 also regulated the expression of PAI-1, an independent prognostic factor in BCa (Ota et al., 2007). As reported in previous study, patients with high PAI-1 level benefit from adjuvant chemotherapy (Kim et al., 2016), indicating that corylin potentially executes its function via the miR-22-3p/ SIRT1/PAI-1 regulatory axis. However, it still needs further validation. In conclusion, corylin sensitized TAMresistant cells to TAM treatment by inhibiting OAS1 expression and modulating the OAS1/miR-22-3p/SIRT1 axis.

\section{Acknowledgements}

Not applicable.

\section{Conflict of Interest}

Authors declare that there is no conflict of interest in this study.

\section{REFERENCES}

Cao L, Xiang G, Liu F, Xu C, Liu J, Meng Q, Lyu S, Wang S, Niu Y (2019) A high AR:ERalpha or PDEF:ERalpha ratio predicts a sub-optimal response to tamoxifen therapy in ERalpha-positive breast cancer. Cancer Chemother Pharmacol 84: 609-620. https://doi, org/10.1007/s00280-019-03891-6

Choi UY, Kang JS, Hwang YS, Kim YJ (2015) Oligoadenylate synthaselike (OASL) proteins: dual functions and associations with diseases. Exp Mol Med 47: e144. https://doi.org/10.1038/emm.2014.110

Hancks DC, Hartley MK, Hagan C, Clark NL, Elde NC (2015) Overlapping patterns of rapid evolution in the nucleic acid sensors cGAS and OAS1 suggest a common mechanism of pathogen antagonism and escape. PLoS Genet 11: e1005203. https://doi.org/10.1371/journal.pgen.1005203

Hovnanian A, Rebouillat D, Mattei MG, Levy ER, Marie I, Monaco AP, Hovanessian AG (1998) The human 2',5'-oligoadenylate synthetase locus is composed of three distinct genes clustered on chromosome $12 \mathrm{q} 24.2$ encoding the $100-, 69-$, and $40-\mathrm{kDa}$ forms. Genomics 52: 267-277. https://doi.org/10.1006/geno.1998.5443

Hultsch S, Kankainen M, Paavolainen L, Kovanen RM, Ikonen E, Kangaspeska S, Pietiainen V, Kallioniemi O (2018) Association of tamoxifen resistance and lipid reprogramming in breast cancer. BMC Cancer 18: 850. https://doi.org/10.1186/s12885-018-4757-z

Ignatiadis M, Sotiriou C (2013) Luminal breast cancer: from biology to treatment. Nat Rev Clin Oncol 10: 494-506. https://doi.org/10.1038/ nrclinonc. 2013.124

Kim EY, Do SI, Hyun K, Park YL, Kim DH, Chae SW, Sohn JH, Park CH (2016) High expression of urokinase-type plasminogen activator is associated with lymph node metastasis of invasive ductal carcinoma of the breast. J Breast Cancer 19: 156-162. https://doi. org/10.4048/jbc.2016.19.2.156

Kristiansen H, Gad HH, Eskildsen-Larsen S, Despres P, Hartmann R (2011) The oligoadenylate synthetase family: an ancient protein fam- ily with multiple antiviral activities. I Interferon Cytokine Res 31: 41-47. https://doi.org/10.1089/jir.2010.0107

Lee JH, Park SJ, Jeong SY, Kim MJ, Jun S, Lee HS, Chang IY, Lim SC, Yoon SP, Yong J, You HJ (2015) MicroRNA-22 suppresses DNA repair and promotes genomic instability through targeting of MDC1. Cancer Res 75: 1298-1310. https://doi.org/10.1158/00085472.CAN-14-2783

Lim E, Winer EP (2011) Adjuvant chemotherapy in luminal breast cancers. Breast 20 (Suppl 3): S128-S131. https://doi.org/10.1016/ S0960-9776(11)70309-5

Livak KJ, Schmittgen TD (2001) Analysis of relative gene expression data using real-time quantitative PCR and the 2(-Delta Delta C(T)) Method. Methods 25: 402-408. https://doi.org/10.1006/ meth.2001.1262

Lu C, Wang H, Chen S, Yang R, Li H, Zhang G (2018) Baicalein inhibits cell growth and increases cisplatin sensitivity of A549 and H460 cells via miR-424-3p and targeting PTEN/PI3K/Akt pathway. J Cell Mol Med 22: 2478-2487. https://doi.org/10.1111/jcmm.13556

Ota H, Akishita M, Eto M, Iijima K, Kaneki M, Ouchi Y (2007) Sirt1 modulates premature senescence-like phenotype in human endothelial cells. J Mol Cell Cardiol 43: 571-579. https://doi.org/10.1016/j. yjmcc.2007.08.008

Peritz T, Zeng F, Kannanayakal TJ, Kilk K, Eiriksdottir E, Langel U, Eberwine J (2006) Immunoprecipitation of mRNA-protein complexes. Nat Protoc 1: 577-580. https://doi.org/10.1038/nprot.2006.82

Qiu G, Jin Z, Chen X, Huang J (2020) Interpretation of guidelines for the diagnosis and treatment of primary liver cancer (2019 edn) in China. Glob Health Med 2: 306-311. https://doi.org/10.35772/ ghm.2020.01051

Siegel RL, Miller KD, Jemal A (2018) Cancer statistics, 2018. CA Cancer J Clin 68: 7-30. https://doi.org/10.3322/caac.21442

Sun R, Liu Z, Han L, Yang Y, Wu F, Jiang Q, Zhang H, Ma R, Miao J, He K, Wang X, Zhou D, Huang C (2019) miR-22 and miR-214 targeting BCL9L inhibit proliferation, metastasis, and epithelialmesenchymal transition by down-regulating Wnt signaling in colon cancer. FASEB J 33: 5411-5424. https://doi.org/10.1096/ fj.201801798RR

Szczerba BM, Castro-Giner F, Vetter M, Krol I, Gkountela S, Landin J, Scheidmann MC, Donato C, Scherrer R, Singer J, Beisel C, Kurzeder C, Heinzelmann-Schwarz V, Rochlitz C, Weber WP, Beerenwinkel N, Aceto N (2019) Neutrophils escort circulating tumour cells to enable cell cycle progression. Nature 566: 553-557. https://doi.org/10.1038/s41586-019-0915-y

Tian Y, Guan Y, Su Y, Yang T, Yu H (2021) TRPM2-AS promotes bladder cancer by targeting miR-22-3p and regulating GINS2 mRNA expression. Onco Targets Ther 14: 1219-1237. https://doi. org/10.2147/OT'T.S282151

Tsuchiya N, Izumiya M, Ogata-Kawata H, Okamoto K, Fujiwara Y, Nakai M, Okabe A, Schetter AJ, Bowman ED, Midorikawa Y, Sugiyama Y, Aburatani H, Harris CC, Nakagama H (2011) Tumor suppressor miR-22 determines p53-dependent cellular fate through post-transcriptional regulation of p21. Cancer Res 71: 4628-4639. https://doi.org/10.1158/0008-5472.CAN-10-2475

Wang B, Wang K, Jin T, Xu Q, He Y, Cui B, Wang Y (2020) NCK1AS1 enhances glioma cell proliferation, radioresistance and chemoresistance via miR-22-3p/IGF1R ceRNA pathway. Biomed Pharmacother 129: 110395. https://doi.org/10.1016/j.biopha.2020.110395

Xu D, Takeshita F, Hino Y, Fukunaga S, Kudo Y, Tamaki A, Matsunaga J, Takahashi RU, Takata T, Shimamoto A, Ochiya T, Tahara $\mathrm{H}$ (2011) miR-22 represses cancer progression by inducing cellular senescence. J Cell Biol 193: 409-424. https://doi.org/10.1083/ jcb.201010100

Yang X, Su W, Li Y, Zhou Z, Zhou Y, Shan H, Han X, Zhang M, Zhang Q, Bai Y, Guo C, Yang S, Beer DG, Chen G (2021) MiR22-3p suppresses cell growth via MET/STAT3 signaling in lung cancer. Am J Transl Res 13: 1221-1232

Zhang Q, Li X, Cui K, Liu C, Wu M, Prochownik EV, Li Y (2020) The MAP3K13-TRIM25-FBXW7alpha axis affects c-Myc protein stability and tumor development. Cell Death Differ 27: 420-433. https://doi.org/10.1038/s41418-019-0363-0

Zhang Y, Yu C (2020) Prognostic characterization of OAS1/OAS2/ OAS3/OASL in breast cancer. BMC Cancer 20: 575. https://doi. org/10.1186/s12885-020-07034-6 